\title{
Interval-Valued Intuitionistic Fuzzy Soft Rough Approximation Operators and Their Applications in Decision Making Problem
}

\author{
Anjan Mukherjee ${ }^{1} \cdot$ Abhik Mukherjee $^{2}$
}

Received: 4 August 2021 / Revised: 4 January 2022 / Accepted: 10 January 2022 /

Published online: 7 February 2022

(c) The Author(s), under exclusive licence to Springer-Verlag GmbH Germany, part of Springer Nature 2022

\begin{abstract}
It has been found that fuzzy sets, rough sets and soft sets are closely related concepts. Many complicated problems in economics, engineering, social sciences, medical science and many other fields involve uncertain data. These problems, which one comes in real life, cannot be solved using classical mathematical methods. There are several well-known theories to describe uncertainty, for instance, fuzzy set theory, rough set theory, and other mathematical tools. But all of these theories have their inherit difficulties as pointed out by D. Molodtsov. In 1999, D. Molodtsov introduced the concept of soft sets, which can be seen as a new mathematical tool for dealing with uncertainties. The concept of rough sets, proposed by Z. Pawlak as a framework for the construction of approximations of concepts. It is a formal tool for modeling and processing insufficient and incomplete information. Zhou and $\mathrm{Wu}$ first proposed the concept of intuitionistic fuzzy rough sets (IFrough sets). The aim of this paper is to introduce the concept of interval-valued intuitionistic fuzzy soft rough sets (IVIFS rough sets). We also investigate some properties of IVIFS rough approximation operators. Some basic operations and properties are studied. Lastly applications have been shown in decision making problems.
\end{abstract}

Keywords Fuzzy set · Rough set · Soft set · Intuitionistic fuzzy rough set · Interval-valued fuzzy rough soft set

Mathematics Subject Classification 03E72 · 90B50 $\cdot 03 \mathrm{E} 75$

\footnotetext{
$凶$ Anjan Mukherjee

mukherjee123anjan@gmail.com

Abhik Mukherjee

abhikhaider82@gmail.com

1 Department of Mathematics, Tripura University, Suryamaninagar, Agartala 799022, India

2 Department of Conservative Dentistry and Endodontics, ITS Dental College, Muradnagar, Delhi-Meerut Road, Ghaziabad 201206, India
} 


\section{Introduction}

In [1], Zhou and Wu first proposed the concept of IFS rough sets. It is the combination of fuzzy soft relation and the intuitionistic fuzzy rough sets. They investigated some properties of IFS rough approximation operators. Moreover, many new rough set models have also been established by combining the Pawlak rough set with other uncertainty theories, Feng et al. [2] provided a framework to combine fuzzy sets, rough sets, and soft sets all together. It gives rise to several interesting new concepts such as rough soft sets, soft rough sets, and soft rough fuzzy sets. The combination of soft set and rough set models was also studied in [3].

In this paper, we propose the notion of interval-valued intuitionistic fuzzy soft rough sets (IVIFS rough sets). We combine a IVIF soft relation with interval-valued intuitionistic fuzzy rough sets. Then we define the upper and lower approximations of any IVIF set on parameter set $E$. IVIFS rough sets can also be exploited to extend many practical applications in real life. Therefore, we propose a novel approach to decision making based on IVIFS rough set theory.

The rest of this paper is organized as follows. In Sect. 2, we review some basic notions related to soft sets, fuzzy soft sets, intuitionistic fuzzy sets, interval-valued intuitionistic fuzzy sets. In Sect. 3, we construct the definition of IVIFS rough sets and investigate some of their interesting properties. We also investigate some properties of IVIFS rough approximation operators. Section 4 is devoted to studying the application of IVIFS rough sets. Section 5 explores knowledge discovering in COVID-19 pandemic. In particular it discusses about the people who affected severely in COVID-19. We use IVIF soft rough operators for determining COVID-19 patients. Some conclusions and outlooks for further research are given in Sect. 6.

Data science is an interdisciplinary field focused on extracting knowledge from data sets, which are typically large (big data), and applying the knowledge and actionable insights from data to solve problems in a wide range of application domains. The field encompasses preparing data for analysis, formulating data science problems, analyzing data, developing data-driven solutions, and presenting findings to inform high-level decisions in a broad range of application domains.

Data Science and related technologies are critical in the fight against pandemics, such as the 2003 severe acute respiratory syndrome coronavirus (SARS-CoV), COVID-19, to enable governments and health managements figure out the best preparation and response. Big data, data mining, machine learning, and a variety of other technologies can be used to swiftly and effectively evaluate data in order to track and regulate COVID-19's spread over the world [4-7]. Big data is currently a hot topic among researchers, engineers, health-care executives, and administrators [8]. Several academics have made extensive use of data mining techniques to uncover hidden information in large datasets [4-7]. The results obtained from the study by Liu et al. [6] can be linked to India and other countries, which will be valuable in understanding the virus transmission patterns among the population of different age-groups. Further we study the references [8-13]. 


\section{Preliminaries}

The following definitions and preliminaries are required in our future work.

Definition 2.1 ([14]) Let $X$ be an ordinary set. A fuzzy subset $\alpha$ in $X$ is the collection of ordered pairs $\left(\mathrm{x}, \mu_{\alpha}(\mathrm{x})\right)$ with $\mathrm{x} \in \mathrm{X}$ and a membership function $\mu_{\alpha}: \mathrm{X} \rightarrow[0,1]$. The value $\mu_{\alpha}(\mathrm{x})$ of $\mathrm{x}$ denotes the degree to which an element $\mathrm{x}$ may be a member of $\alpha$. Thus a fuzzy subset $\alpha$ of $X$ is denoted by $\alpha=\left\{\left(x, \mu_{\alpha}(x)\right): x \in X\right\}$ where $\mu_{\alpha}(x)$ $=1$, indicates strictly the containment of the element $\mathrm{x}$ in $\alpha$ (full membership) and $\mu_{\alpha}(\mathrm{x})=0$ denotes that $\mathrm{x}$ does not belong to $\alpha$ (non-membership). Thus an ordinary set is a special case of fuzzy set with a membership function which is reduced to a characteristic function. Because of these generalities the fuzzy set theory has a wider scope of applicability than the ordinary set theory in solving real problems.

A fuzzy set $\alpha$ can also be represented in the following way $\alpha=\left\{x / \mu_{\alpha}(x), \forall x \in\right.$ $\mathrm{X}\}$ or $\alpha=\left\{\left(\mathrm{x}, \mu_{\alpha}(\mathrm{x})\right): \mathrm{x} \in \mathrm{X}\right\}$ The set of all fuzzy subset on $\mathrm{X}$ is denoted by $\mathrm{I}^{\mathrm{X}}$. After the introduction of concept of fuzzy sets by Zadeh, several researches generalized the notion of the fuzzy set. The idea of "intuitionistic fuzzy set "was first published by Atanassov in 1983.

Definition 2.2 ([15]) Let a set $\mathrm{E}$ be fixed. An intuitionistic fuzzy set or IFS 'A' in $\mathrm{E}$ is an object having the form $\mathrm{A}=\left\{\left(\mathrm{x}, \mu_{\mathrm{A}}(\mathrm{x}), \nu_{\mathrm{A}}(\mathrm{x}): \mathrm{x} \in \mathrm{E}\right)\right\}$ where the function $\mu_{\mathrm{A}}: \mathrm{E} \rightarrow$ $\mathrm{I}=[0,1] \& v_{\mathrm{A}}: \mathrm{E} \rightarrow \mathrm{I}=[0,1]$ define the degree of membership and non-membership respectively of the element $\mathrm{x} \in \mathrm{E}$ to the set $\mathrm{A} \&$ for every $\mathrm{x} \in \mathrm{E}, 0 \leq \mu_{\mathrm{A}}(\mathrm{x})+v_{\mathrm{A}}(\mathrm{x})$ $\leq 1$. The rest part $\pi_{A}(x)=1-\mu_{A}(x)-v_{A}(x)$ is called the indeterministic part of $\mathrm{x}$ and $0 \leq \pi_{\mathrm{A}}(\mathrm{x}) \leq 1$.

Definition 2.3 ([16] An interval valued fuzzy set) A over a universe set $\mathrm{U}$ is defined as the object of the form $A=\left\{\left(x, \mu_{A}(x): x \in U\right)\right\}$, where $\mu_{A}(x): U \rightarrow \operatorname{Int}([0,1])$ is a function, where Int $([0,1])$ denotes the set of all closed sub intervals of $[0,1]$.

Definition 2.4 ([17] An interval valued intuitionistic fuzzy set) A over a universe set U is defined as the object of the form $\left.A=\left\{\left\langle x, \mu_{A}(x), \gamma_{A}(x)\right\rangle: x \in U\right)\right\}$, where $\mu_{A}(x)$ : $\mathrm{U} \rightarrow$ Int $([0,1])$ and $\gamma_{\mathrm{A}}(\mathrm{x}): \mathrm{U} \rightarrow$ Int $([0,1])$ are functions such that the condition: $\forall \mathrm{x}$ $\in \mathrm{U}, \sup \mu_{\mathrm{A}}(\mathrm{x})+\sup \gamma_{\mathrm{A}}(\mathrm{x}) \leq 1$ is satisfied.

The class of all interval valued intuitionistic fuzzy soft sets on $\mathrm{U}$ is denoted by IVIFS $^{\mathrm{U}}$. For an arbitrary set $\mathrm{A} \subseteq[0,1]$, we use $\underline{\mathrm{A}}=\inf \mathrm{A}$ and $\bar{A}=\sup \mathrm{A}$.

Definition 2.5 ([18, 19]) Let $\mathrm{R}$ be an equivalence relation on the universal set $\mathrm{U}$. Then the pair $(\mathrm{U}, \mathrm{R})$ is called a Pawlak approximation space. An equivalence class of $\mathrm{R}$ containing $\mathrm{x}$ will be denoted by $[\mathrm{x}]_{\mathrm{R}}$. Now for $\mathrm{X} \subseteq \mathrm{U}$, the lower and upper approximation of $\mathrm{X}$ with respect to $(\mathrm{U}, \mathrm{R})$ are denoted by respectively $\mathrm{R} * \mathrm{X} \& \mathrm{R} * \mathrm{X}$ and are defined by

$$
\begin{gathered}
\mathrm{R}_{*} \mathrm{X}=\left\{\mathrm{x} \in \mathrm{U}:[\mathrm{x}]_{\mathrm{R}} \subseteq \mathrm{X}\right\} \text { or } \cup\left\{[\mathrm{x}]_{\mathrm{R}}:[\mathrm{x}]_{\mathrm{R}} \subseteq \mathrm{X}\right\}, \quad \mathrm{x} \in \mathrm{U} \\
\mathrm{R} * \mathrm{X}=\left\{\mathrm{x} \in \mathrm{U}:[\mathrm{x}]_{\mathrm{R}} \cap \mathrm{X} \neq \phi\right\} \text { or } \cup\left\{[\mathrm{x}]_{\mathrm{R}}:[\mathrm{x}]_{\mathrm{R}} \cap \mathrm{X} \neq \phi\right\}, \quad \mathrm{x} \in \mathrm{U}
\end{gathered}
$$

Now if $\mathrm{R} * \mathrm{X}=\mathrm{R} * \mathrm{X}$, then $\mathrm{X}$ is called definable otherwise $\mathrm{X}$ is called a Rough set. 
Definition 2.6 ([20-24]) Let $U$ be an initial universe and $E$ be a set of parameters. Let $\mathrm{P}(\mathrm{U})$ denotes the power set of $\mathrm{U}$ and $\mathrm{A} \subseteq \mathrm{E}$. Then the pair $(\mathrm{F}, \mathrm{A})$ is called a soft set over $\mathrm{U}$, where $\mathrm{F}$ is a mapping given by $\mathrm{F}: \mathrm{A} \rightarrow \mathrm{P}(\mathrm{U})$.

For any $\varepsilon \in \mathrm{A}, \mathrm{F}(\varepsilon) \subseteq \mathrm{U}$ may be considered as the set of $\varepsilon$-approximate elements of the soft set (F, A).

Definition 2.7 ([25, 26]) Let $U$ be an initial universe and $E$ be a set of parameters. Let $\mathrm{I}^{\mathrm{U}}$ be the set of all fuzzy subsets of $\mathrm{U}$ and $\mathrm{A} \subseteq \mathrm{E}$. Then the pair $(\mathrm{F}, \mathrm{A})$ is called a fuzzy soft set over $\mathrm{U}$, where $\mathrm{F}$ is a mapping given by $\mathrm{F}: \mathrm{A} \rightarrow \mathrm{I}^{\mathrm{U}}$.

For any $\varepsilon \in A, F(\varepsilon)$ is a fuzzy subset of $U$. Let us denote the membership degree that object $\mathrm{x}$ holds parameter $\varepsilon$ by $\mu_{\mathrm{F}(\varepsilon)}(\mathrm{x})$, where $\mathrm{x} \in \mathrm{U}$ and $\varepsilon \in \mathrm{A}$. Then $\mathrm{F}(\varepsilon)$ can be written as a fuzzy set such that $F(\varepsilon)=\left\{\left(x, \mu_{F(\varepsilon)}(x)\right): x \in U\right\}$.

Definition 2.8 ([27]) Let $U$ be an initial universe and $E$ be a set of parameters. Let I $\mathrm{U}$ be the set of all fuzzy subsets of $\mathrm{U}$ and $\mathrm{A} \subseteq \mathrm{E}$. Now, $\mathrm{F}: \mathrm{A} \rightarrow \mathrm{I}^{\mathrm{U}}$, and $\alpha$ be a fuzzy subset of $\mathrm{A}$ i.e. $\alpha: \mathrm{A} \rightarrow \mathrm{I}=[0,1]$. Let $\mathrm{F}_{\alpha}$ be a mapping $\mathrm{F}_{\alpha}: \mathrm{A} \rightarrow \mathrm{I}^{\mathrm{U}} \times \mathrm{I}$ defined as follows: $F_{\alpha}(\varepsilon)=(F(\varepsilon), \alpha(\varepsilon))$ then $F_{\alpha}$ is called generalised fuzzy soft set over the soft universe (U, A).

For any $\varepsilon \in A, F(\varepsilon)$ is a fuzzy subset of $U$. Let us denote the membership degree that object $\mathrm{x}$ holds parameter $\varepsilon$ by $\mu_{\mathrm{F}(\varepsilon)}(\mathrm{x})$, where $\mathrm{x} \in \mathrm{U}$ and $\varepsilon \in \mathrm{A}$. Then $\mathrm{F}(\varepsilon)$ can be written as a fuzzy set such that $\mathrm{F}(\varepsilon)=\left\{\left(\mathrm{x}, \mu_{\mathrm{F}(\varepsilon)}(\mathrm{x})\right): \mathrm{x} \in \mathrm{U}\right\}$.

Definition 2.9 ([28]) Let $U$ be an initial universe and $E$ be a set of parameters. Let I $\mathrm{F}^{\mathrm{U}}$ be the set of all intuitionistic fuzzy subsets of $\mathrm{U}$ and $\mathrm{A} \subseteq \mathrm{E}$. Then the pair $(\mathrm{F}, \mathrm{A})$ is called an intuitionistic fuzzy soft set over $\mathrm{U}$, where $\mathrm{F}$ is a mapping given by $\mathrm{F}: \mathrm{A} \rightarrow \mathrm{I}$ $\mathrm{F}^{\mathrm{U}}$.

For any $\varepsilon \in A, F(\varepsilon)$ is an intuitionistic fuzzy subset of $U$. Let us denote $\mu_{F(\varepsilon)}(x)$ and $\gamma_{\mathrm{F}(\varepsilon)}(\mathrm{x})$ by the membership degree \& non-membership degree respectively that object $\mathrm{x}$ holds parameter $\varepsilon$, where $\mathrm{x} \in \mathrm{U}$ and $\varepsilon \in \mathrm{A}$. Then $\mathrm{F}(\varepsilon)$ can be written as an intuitionistic fuzzy set such that $\mathrm{F}(\varepsilon)=\left\{\left(\mathrm{x}, \mu_{\mathrm{F}(\varepsilon)}(\mathrm{x}), \gamma_{\mathrm{F}(\varepsilon)}(\mathrm{x})\right): \mathrm{x} \in \mathrm{U}\right\}$.

Now before we introduce the notion of the interval valued intuitionistic fuzzy soft sets, let us give the concept of interval valued intuitionistic fuzzy set which was first introduced by Atanassov and Gargov [17]. Actually an interval valued intuitionistic fuzzy set is characterized by an interval-valued membership degree and an intervalvalued non-membership degree.

Definition 2.10 ([17] An interval valued intuitionistic fuzzy set (IVIFS for short)) A on an universe set $U$ is defined as the object of the form $A=\left\{\left\langle x, \mu_{A}(x), \gamma_{A}(x)\right\rangle\right.$ : $\mathrm{x} \in \mathrm{U}\}$, where $\mu_{\mathrm{A}}: \mathrm{U} \rightarrow \operatorname{Int}([0,1])$ and $\gamma_{\mathrm{A}}: \mathrm{U} \rightarrow$ Int $([0,1])$ are functions such that the condition: $\forall \mathrm{x} \in \mathrm{U}, \sup \mu_{\mathrm{A}}(\mathrm{x})+\sup \gamma_{\mathrm{A}}(\mathrm{x}) \leq 1$ is satisfied (where $\operatorname{Int}[0,1]$ is the set of all closed intervals of $[0,1])$.

We denote the class of all interval valued intuitionistic fuzzy sets on U by IVIFS ${ }^{\mathrm{U}}$.

The union and intersection of the interval valued intuitionistic fuzzy sets are defined as follows:

Let $\mathrm{A}, \mathrm{B} \in \mathrm{IVIFS}^{\mathrm{U}}$. Then

- the union of $\mathrm{A}$ and $\mathrm{B}$ is denoted by $\mathrm{A} \cup \mathrm{B}$ where

$A \cup B=\left\{\left(x,\left[\max \left(\inf \mu_{A}(x), \inf \mu_{B}(x)\right), \max \left(\sup \mu_{A}(x), \sup \mu_{B}(x)\right)\right],\left[\min \left(\inf \gamma_{A}(x)\right.\right.\right.\right.$, $\left.\left.\left.\left.\inf \gamma_{B}(x)\right), \min \left(\sup \gamma_{A}(x), \sup \gamma_{B}(x)\right)\right]\right): x \in U\right\}$. 
- the intersection of $\mathrm{A}$ and $\mathrm{B}$ is denoted by $\mathrm{A} \cap \mathrm{B}$ where

$A \cap B=\left\{\left(x,\left[\min \left(\inf \mu_{A}(x), \inf \mu_{B}(x)\right), \min \left(\sup \mu_{A}(x), \sup \mu_{B}(x)\right)\right],\left[\max \left(\inf \gamma_{A}(x)\right.\right.\right.\right.$, $\left.\left.\left.\left.\inf \gamma_{B}(x)\right), \max \left(\sup \gamma_{A}(x), \sup \gamma_{B}(x)\right)\right]\right): x \in U\right\}$.

Atanassov and Gargov showed in [17] that $\mathrm{A} \cup \mathrm{B}$ and $\mathrm{A} \cap \mathrm{B}$ are again IVIFSs.

Definition 2.11 ([2]) Let $U$ be an universe set and $\mathrm{E}$ be a set of parameters. Let IVIFS ${ }^{\mathrm{U}}$ be the set of all interval valued intuitionistic fuzzy sets on $U$ and $A \subseteq E$. Then the pair (F, A) is called an interval valued intuitionistic fuzzy soft set (IVIFS set) over U, where $\mathrm{F}$ is a mapping given by $\mathrm{F}: \mathrm{A} \rightarrow$ IVIFS $^{\mathrm{U}}$.

In other words, an interval valued intuitionistic fuzzy soft set is a parameterized family of interval valued intuitionistic fuzzy subsets of $U$. For any parameter e $\in A, F(e)$ can be written as an interval valued intuitionistic fuzzy set such that $F(e)=\left\{\left(x, \mu_{F(e)}(x)\right.\right.$, $\left.\left.\gamma_{\mathrm{F}(\mathrm{e})}(\mathrm{x})\right): \mathrm{x} \in \mathrm{U}\right\}$ where $\mu_{\mathrm{F}(\mathrm{e})}(\mathrm{x})$ is the interval valued fuzzy membership degree that object $\mathrm{x}$ holds parameter $\mathrm{e}$ and $\gamma_{\mathrm{F}(\mathrm{e})}(\mathrm{x})$ is the interval valued fuzzy membership degree that object $\mathrm{x}$ doesn't hold parameter e.

Definition 2.12 ([1, 3, 29, 30]) Let $U$ be an initial universe set and let $E$ be a universe set of parameters. For an arbitrary IF soft relation $R$ over $U \times E$, the pair $(U, E, R)$ is called a fuzzy soft approximation space. For any $A \in \operatorname{IF}(E)$, the upper and lower IF soft approximations of $A$ with respect to $(U, E, R)$, denoted by $\bar{R}(A)$ and $\underline{R}(A)$ respectively as follows:

$$
\begin{aligned}
& \bar{R}(A)=\left\{<u, \mu_{\bar{R}(A)}(u), \gamma_{\bar{R}(A)}(u)>\mid u \in U\right\} \\
& \underline{R}(A)=\left\{<u, \mu_{\underline{R}(A)}(u), \gamma_{\underline{R}(A)}(u)>\mid u \in U\right\}
\end{aligned}
$$

where $\mu_{\bar{R}(A)}(u)=\vee_{x \in E}\left[\mu_{R}(u, x) \wedge \mu_{A}(x)\right]$

$$
\begin{aligned}
& \gamma_{\bar{R}(A)}(u)=\wedge_{x \in E}\left[1-\mu_{R}(u, x) \vee \gamma_{A}(x)\right] \\
& \mu_{\underline{R}(A)}(u)=\wedge_{x \in E}\left[1-\mu_{R}(u, x) \vee \mu_{A}(x)\right] \\
& \gamma_{\underline{R}(A)}(u)=\vee_{x \in E}\left[1-\mu_{R}(u, x) \wedge \gamma_{A}(x)\right]
\end{aligned}
$$

The pair $(\bar{R}(A), \underline{R}(A))$ is an intuitionistic fuzzy soft rough set of A with respect to (U, E, R).

\section{Interval-Valued Intuitionistic Fuzzy Soft Rough Sets}

In [1], Zhou and Wu first presented the concept of intuitionistic fuzzy rough sets. In this section, we introduce the definition of interval-valued intuitionistic fuzzy soft rough sets. It is the combination of IVIF soft relation with the interval-valued intuitionistic fuzzy rough sets. 
We also investigate some properties of interval-valued intuitionistic fuzzy soft rough approximation operators.

Definition 3.1 Let $U$ be an initial universe set and let $E$ be a universe set of parameters. For an arbitrary IVIF soft relation $R$ over $U \times E$, the pair $(U, E, R)$ is called a IVIF soft approximation space. For any $A \in \operatorname{IVIF}(E)$, we define the upper and lower intervavalued intuitionistic fuzzy soft approximations of $A$ with respect to $(U, E, R)$, denoted by $\bar{R}(A)$ and $\underline{R}(A)$ respectively as follows:

$$
\bar{R}(A)=\left\{<u, \mu_{\bar{R}(A)}(u), \gamma_{\bar{R}(A)}(u)>\mid u \in U\right\}
$$

where $\mu_{\bar{R}(A)}(u)=\left[\mu_{\bar{R}(A)}^{l}(u), \mu_{\bar{R}(A)}^{r}(u)\right]$, and $\gamma_{\bar{R}(A)}(u)=\left[\gamma_{\bar{R}(A)}^{l}(u), \gamma_{\bar{R}(A)}^{r}(u)\right]$. $\underline{R}(A)=\left\{<u, \mu_{R(A)}(u), \gamma_{R(A)}(u)>\mid u \in U\right\}$, where $\mu_{\underline{R}(A)}(u)=\left[\mu_{\underline{R}(A)}^{l}(u), \mu_{\underline{R}(A)}^{r}(u)\right]$ and $\gamma_{\underline{R}(A)}(u)=\left[\gamma_{\underline{R}(A)}^{l}(u), \gamma_{\underline{R}(A)}^{r}(u)\right]$.

Therefore, $\bar{R}(A)=\left\{<u,\left[\mu_{\bar{R}(A)}^{l}(u), \mu_{\bar{R}(A)}^{r}(u)\right],\left[\gamma_{\bar{R}(A)}^{l}(u), \gamma_{\bar{R}(A)}^{r}(u)\right]>\mid u \in U\right\}$

$$
\underline{R}(A)=\left\{<u,\left[\mu_{\underline{R}(A)}^{l}(u), \mu_{\underline{R}(A)}^{r}(u)\right],\left[\gamma_{\underline{R}(A)}^{l}(u), \gamma_{\underline{R}(A)}^{r}(u)\right]>\mid u \in U\right\}
$$

where $\mu_{\bar{R}(A)}^{l}(u)=\vee_{x \in E}\left[\mu_{R}^{l}(u, x) \wedge \mu_{A}^{l}(x)\right]$

$$
\begin{aligned}
& \mu_{\bar{R}(A)}^{r}(u)=\vee_{x \in E}\left[\mu_{R}^{r}(u, x) \wedge \mu_{A}^{r}(x)\right] \\
& \gamma_{\frac{R}{R}(A)}^{l}(u)=\wedge_{x \in E}\left[\gamma_{R}^{l}(u, x) \vee \gamma_{A}^{l}(x)\right] \\
& \gamma_{\bar{R}(A)}^{r}(u)=\wedge_{x \in E}\left[\gamma_{R}^{r}(u, x) \vee \gamma_{A}^{r}(x)\right] \\
& \mu_{\underline{R}(A)}^{l}(u)=\wedge_{x \in E}\left[\mu_{R}^{l}(u, x) \vee \mu_{A}^{l}(x)\right] \\
& \mu_{\underline{R}(A)}^{r}(u)=\wedge_{x \in E}\left[\mu_{R}^{r}(u, x) \vee \mu_{A}^{r}(x)\right] \\
& \gamma_{\underline{R}(A)}^{l}(u)=\vee_{x \in E}\left[\gamma_{R}^{l}(u, x) \wedge \gamma_{A}^{l}(x)\right] \\
& \gamma_{\underline{R}(A)}^{r}(u)=\vee_{x \in E}\left[\gamma_{R}^{r}(u, x) \wedge \gamma_{A}^{r}(x)\right]
\end{aligned}
$$

The pair $(\bar{R}(A), \underline{R}(A))$ is an interval-valued intuitionistic fuzzy soft rough set (IVIF soft rough set) of A with respect to (U, E, R). 
Theorem 3.2. $\bar{R}(A)$ and $\underline{R}(A) \in \operatorname{IVIF}(\mathrm{u})$

\section{Proof}

$$
\begin{aligned}
& \sup \mu_{\bar{R}(A)}(u)+\sup _{\bar{R}(A)}(u) \\
& =\mu_{\bar{R}(A)}^{r}(u)+\gamma_{\bar{R}(A)}^{r}(u) \\
& =\vee_{x \in E}\left[\mu_{R}^{r}(u, x) \wedge \mu_{A}^{r}(x)\right]+\wedge_{x \in E}\left[\gamma_{R}^{r}(u, x) \vee \gamma_{A}^{r}(x)\right] \\
& \leq \vee_{x \in E}\left[\mu_{R}^{r}(u, x) \wedge \mu_{A}^{r}(x)\right]+\wedge_{x \in E}\left[1-\mu_{R}^{r}(u, x) \vee \gamma_{A}^{r}(x)\right] \\
& \text { since } \mu_{R}^{r}(u, x)+\gamma_{R}^{r}(u, x) \leq 1 \\
& \quad=\vee_{x \in E}\left[\mu_{R}^{r}(u, x) \wedge \mu_{A}^{r}(x)\right]+1-\vee_{x \in E}\left[\mu_{R}^{r}(u, x) \wedge \gamma_{A}^{r}(x)\right]=1
\end{aligned}
$$

Hence, $\bar{R}(A) \in \operatorname{IVIF}(\mathrm{U})$. Similarly, we have $\underline{R}(A)) \in \operatorname{IVIF}(\mathrm{U})$. We call $\bar{R}, \underline{R}$ : $\operatorname{IVIF}(\mathrm{E}) \rightarrow \operatorname{IVIF}(\mathrm{U})$.

The upper and lower IVIF soft rough approximation operations respectively.

Example 3.3 Suppose that, $U=\left\{u_{1}, u_{2}, u_{3}, u_{4}\right\}$, is the set of four houses under consideration of a decision maker to purchase. Let $\mathrm{E}$ be a parameter set where $\mathrm{E}=$ $\left\{\mathrm{e}_{1}, \mathrm{e}_{2}, \mathrm{e}_{3}\right\}=$ expensive, beautiful, location $\}$. Mr. X wants to buy the house which qualities with the parameters of $\mathrm{E}$.

Assume that Mr. decides the "attractiveness of the house' by constructing an IVIF fuzzy soft set $(\mathrm{F}, \mathrm{E})$ which is an IVIF soft relation $\mathrm{R}$ from $\mathrm{U}$ to $\mathrm{E}$. It is presented by the table:

\begin{tabular}{llll}
\hline $\mathrm{R}$ & $\mathrm{e}_{1}$ & $\mathrm{e}_{2}$ & $\mathrm{e}_{3}$ \\
\hline $\mathrm{u}_{1}$ & {$[0.5,0.7],[0.1,0.3]$} & {$[0,0.1],[0.5,0.8]$} & {$[0.3,0.5],[0.2,0.4]$} \\
$\mathrm{u}_{2}$ & {$[0.1,0.2],[0.4,0.6]$} & {$[0.2,0.5],[0.3,0.4]$} & {$[0.5,0.8],[0.1,0.2]$} \\
$\mathrm{u}_{3}$ & {$[0.1,0.3],[0.4,0.6]$} & {$[0.2,0.3],[0.3,0.6]$} & {$[0,0.1],[0.5,0.8]$} \\
$\mathrm{u}_{4}$ & {$[0.2,0.5],[0.1,0.4]$} & {$[0.3,0.5],[0.2,0.4]$} & {$[0.1,0.2],[0.4,0.7]$} \\
\hline
\end{tabular}

Now suppose that Mr. X gives the optimum normal decision object $\mathrm{A}$ which is an IVIF subset defined as $A=\left\{\left\langle\mathrm{e}_{1},[0.4,0.6],[0.1,0.3]\right\rangle,\left\langle\mathrm{e}_{2},[0.1,0.4],[0.3,0.6]\right\rangle\right.$ $\left.,<\mathrm{e}_{3},[0,0.1],[0.5,0.8]>\right\}$.

By virtue of Definition 3.1

$$
\begin{gathered}
\mu_{\bar{R}(A)}^{l}\left(u_{1}\right)=\vee\{(0.5 \wedge 0.4),(0 \wedge 0.1),(0.3 \wedge 0)\}=\vee\{0.4,0,0\}=0.4 \\
\mu_{\bar{R}(A)}^{r}\left(u_{1}\right)=\vee\{(0.7 \wedge 0.6),(0.1 \wedge 0.4),(0.5 \wedge 0.1)\}=\vee\{0.6,0.1,0.1\}=0.6 \\
\gamma_{\frac{R}{(A)}}^{l}\left(u_{1}\right)=\wedge\{(0.1 \vee 0.1),(0.5 \vee 0.3),(0.2 \vee 0.5)\}=\wedge\{0.1,0.5,0.5\}=0.1
\end{gathered}
$$




$$
\begin{gathered}
\gamma_{\frac{R}{R}(A)}^{r}\left(u_{1}\right)=\wedge\{(0.3 \vee 0.3),(0.8 \vee 0.6),(0.4 \vee 0.8)\}=\wedge\{0.3,0.8,0.8\}=0.3 \\
\mu_{\underline{R}(A)}^{l}\left(u_{1}\right)=\wedge\{(0.1 \vee 0.4),(0.5 \vee 0.1),(0.2 \vee 0)\}=\wedge\{0.4,0.5,0.2\}=0.2 \\
\mu_{\underline{R}(A)}^{r}\left(u_{1}\right)=\wedge\{(0.3 \vee 0.6),(0.8 \vee 0.4),(0.4 \vee 0.4)\}=\wedge\{0.6,0.8,0.4\}=0.4 \\
\gamma_{\underline{R}(A)}^{l}\left(u_{1}\right)=\vee\{(0.5 \wedge 0.1),(0 \wedge 0.3),(0.3 \wedge 0.5)\}=\vee\{0.1,0,0.3\}=0.3 \\
\gamma_{\underline{R}(A)}^{r}\left(u_{1}\right)=\vee\{(0.7 \wedge 0.3),(0.1 \wedge 0.6),(0.5 \wedge 0.8)\}=\vee\{0.3,0.1,0.5\}=0.5
\end{gathered}
$$

\begin{tabular}{ll}
\hline$\mu_{\bar{R}(A)}^{l}\left(u_{2}\right)=0.1$ & $\gamma_{\bar{R}(A)}^{l}\left(u_{2}\right)=0.3$ \\
$\mu_{\bar{R}(A)}^{r}\left(u_{2}\right)=0.4$ & $\gamma_{\bar{R}(A)}^{r}\left(u_{2}\right)=0.6$ \\
$\mu_{\underline{R}(A)}^{l}\left(u_{2}\right)=0.1$ & $\gamma_{\underline{R}(A)}^{l}\left(u_{2}\right)=0.5$ \\
$\mu_{\underline{R}(A)}^{r}\left(u_{2}\right)=0.2$ & $\gamma_{\underline{R}(A)}^{r}\left(u_{2}\right)=0.8$ \\
$\mu_{\bar{R}(A)}^{l}\left(u_{3}\right)=0.1$ & $\gamma_{\bar{R}(A)}^{l}\left(u_{3}\right)=0.3$ \\
$\mu_{\bar{R}(A)}^{r}\left(u_{3}\right)=0.3$ & $\gamma_{\frac{r}{R}(A)}\left(u_{3}\right)=0.6$ \\
$\mu_{\underline{R}(A)}^{l}\left(u_{3}\right)=0.3$ & $\gamma_{\underline{R}(A)}^{l}\left(u_{3}\right)=0.2$ \\
$\mu_{\underline{R}(A)}^{r}\left(u_{3}\right)=0.6$ & $\gamma_{\underline{R}(A)}^{r}\left(u_{3}\right)=0.3$ \\
$\mu_{\bar{R}(A)}^{l}\left(u_{4}\right)=0.2$ & $\gamma_{\bar{R}(A)}^{l}\left(u_{4}\right)=0.1$ \\
$\mu_{\bar{R}(A)}^{r}\left(u_{4}\right)=0.5$ & $\gamma_{\bar{R}(A)}^{r}\left(u_{4}\right)=0.4$ \\
$\mu_{\underline{R}(A)}^{l}\left(u_{4}\right)=0.2$ & $\gamma_{\underline{R}(A)}^{l}\left(u_{4}\right)=0.3$ \\
$\mu_{\underline{R}(A)}^{r}\left(u_{4}\right)=0.4$ & $\gamma_{\underline{R}(A)}^{r}\left(u_{4}\right)=0.5$ \\
\hline
\end{tabular}

Thus $\bar{R}(A)=\left\{<\mathrm{u}_{1},[0.4,0.6],[0.1,0.3]>,<\mathrm{u}_{2},[0.1,0.4],[0.3,0.6]\right\rangle,<\mathrm{u}_{3},[0.1$, $\left.0.3],[0.3,0.6]>,<\mathrm{u}_{4},[0.2,0.5],[0.1,0.4]>\right\}$.

$\left.\& \underline{R}(A)=\left\{<\mathrm{u}_{1},[0.2,0.4],[0.3,0.5]\right\rangle,<\mathrm{u}_{2},[0.1,0.2],[0.5,0.8]\right\rangle,<\mathrm{u}_{3},[0.3$, $\left.0.6],[0.2,0.3]>,<\mathrm{u}_{4},[0.2,0.4],[0.3,0.5]>\right\}$.

\section{Application of IVIF Soft Rough Approximation Operators in Decision Making Problem}

In this article we first adopt the ring sum operation and ring product operation in the following way:

4.1. $\mathrm{H}=\bar{R}(A) \oplus \underline{R}(A)$

$$
=\left\{<u,\left[\left(\mu_{\bar{R}(A)}^{l}(u)+\mu_{\underline{R}(A)}^{l}(u)-\left(\mu_{\bar{R}(A)}^{l}(u) \cdot \mu_{\underline{R}(A)}^{l}(u)\right),\left(\mu_{\bar{R}(A)}^{r}(u)+\mu_{\underline{R}(A)}^{r}(u)\right.\right.\right.\right.
$$




$$
\begin{aligned}
& \left.-\left(\mu_{\bar{R}(A)}^{r}(u) \cdot \mu_{\underline{R}(A)}^{r}(u)\right)\right], \\
& {\left[\left(\gamma_{\bar{R}(A)}^{l}(u) \cdot \gamma_{\underline{R}(A)}^{l}(u), \gamma_{\bar{R}(A)}^{r}(u) \cdot \gamma_{\underline{R}(A)}^{r}(u)\right] \mid u \in U\right\}}
\end{aligned}
$$

4.2. $\mathrm{G}=\bar{R}(A) \otimes \underline{R}(A)$

$$
\begin{aligned}
= & \left\{<u,\left[\left(\mu_{\bar{R}(A)}^{l}(u) \cdot \mu_{\underline{R}(A)}^{l}(u)\right),\left(\mu_{\bar{R}(A)}^{r}(u), \mu_{\underline{R}(A)}^{r}(u)\right)\right],\right. \\
& {\left.\left[\left(\gamma_{\bar{R}(A)}^{l}(u)+\gamma_{\underline{R}(A)}^{l}(u)-\gamma_{\bar{R}(A)}^{l}(u) \cdot \gamma_{\underline{R}(A)}^{l}(u)\right),\left(\gamma_{\bar{R}(A)}^{r}(u), \gamma_{\underline{R}(A)}^{r}(u)\right)\right] \mid u \in U\right\} }
\end{aligned}
$$

Thus from Example 3.3.

$$
\bar{R}(A) \oplus \underline{R}(A)
$$

$=\left\{<\mathrm{u}_{1},[0.52,0.76],[0.03,0.15]\right\rangle,<\mathrm{u}_{2},[0.19,0.52],[0.15,0.48]>,<\mathrm{u}_{3},[0.37$, $\left.0.72],[0.06,0.18]>,<\mathrm{u}_{4},[0.36,0.70],[0.03,0.2]>\right\}$.

Now,

$$
\bar{R}(A) \otimes \underline{R}(A)
$$

$=\left\{<\mathrm{u}_{1},[0.08,0.24],[0.37,0.65]>,<\mathrm{u}_{2},[0.02,0.08],[0.65,0.92]>,<\mathrm{u}_{3},[0.03\right.$, $\left.0.18],[0.44,0.72]>,<\mathrm{u}_{4},[0.04,0.2],[0.37,0.7]>\right\}$.

We observe that $\bar{R}(A) \oplus \underline{R}(A) \& \bar{R}(A) \otimes \underline{R}(A)$ are both IVIF subsets.

4.3. Consider the Example 3.3 we have computed IVIF soft rough approximation operators $\bar{R}(A)$ and $\underline{R}(A)$ of the optimal normal decision object A. In Sect. 4 (article 4.1 and 4.2) we define and calculate $\mathrm{H}=\bar{R}(A) \oplus \underline{R}(A)$ and $\mathrm{G}=\bar{R}(A) \otimes \underline{R}(A)$. The ring sum operation and ring product operation as follows:

Here,

$$
\begin{aligned}
\bar{R}(A) \oplus \underline{R}(A)= & \left\{<\mathrm{u}_{1},[0.52,0.76],[0.03,0.15]>,<\mathrm{u}_{2},[0.19,0.52],[0.15,0.48]>\right. \\
& \left.<\mathrm{u}_{3},[0.37,0.72],[0.06,0.18]>,<\mathrm{u}_{4},[0.36,0.70],[0.03,0.2]>\right\}
\end{aligned}
$$

Obviously the optimal decision is $\mathrm{u}_{1}$ (since the max of the membership value is 0.76 (which is the max. of the others) and max of the non-membership value is 0.15 ( which is the min. of the others). Thus Mr. X will purchase the house $\mathrm{u}_{1}$.

Now, we consider the ring product operation.

Here,

$$
\begin{aligned}
\bar{R}(A) \otimes \underline{R}(A) & =\left\{<\mathrm{u}_{1},[0.08,0.24],[0.37,0.65]>,<\mathrm{u}_{2},[0.02,0.08],[0.65,0.92]>\right. \\
& \left.<\mathrm{u}_{3},[0.03,0.18],[0.44,0.72]>,<\mathrm{u}_{4},[0.04,0.2],[0.37,0.7]>\right\}
\end{aligned}
$$

It is noted that the optimal decision is still $\mathrm{u}_{1}$ (since the max of the membership value is 0.24 (which is the max. of the others) and max of the non-membership value is 0.65 (which is the min. of the others). Hence the Mr. X will purchase the house $u_{1}$. 


\section{4.}

(i) $\bar{R}(A)=\sim \underline{R}(\sim A)$

(ii) $\underline{R}(A)=\sim \overline{\bar{R}}(\sim A)$

where $\sim \mathrm{A}$ is the complement of $\mathrm{A}$.

Proof From definition we have, $\bar{R}(A)=\left\{<u\right.$, [ $\left.\mu_{\bar{R}(A)}^{l}(u), \mu_{\bar{R}(A)}^{r}(u)\right],\left[\gamma_{\bar{R}(A)}^{l}(u)\right.$, $\left.\left.\gamma_{\bar{R}(A)}^{r}(u)\right]>\mid u \in U\right\}$.

$$
\begin{gathered}
=\left\{<u,\left[\left(\vee_{x \in E}\left[\mu_{R}^{l}(u, x) \wedge \mu_{A}^{l}(x)\right]\right),\left(\vee_{x \in E}\left[\mu_{R}^{r}(u, x) \wedge \mu_{A}^{r}(x)\right]\right)\right],\right. \\
\left.\left[\left(\wedge_{x \in E}\left[\gamma_{R}^{l}(u, x) \vee \gamma_{A}^{l}(x)\right]\right),\left(\wedge_{x \in E}\left[\gamma_{R}^{r}(u, x) \vee \gamma_{A}^{r}(x)\right]\right)\right]>\mid u \in U\right\}
\end{gathered}
$$

$$
\begin{aligned}
\underline{\sim R}(\sim A)= & \left\{<u,\left[\gamma_{\underline{R}(\sim A)}^{l}(u), \gamma_{\underline{R}(\sim A)}^{r}(u)\right],\left[\mu_{\underline{R}(\sim A)}^{l}(u), \mu_{\underline{R}(\sim A)}^{r}(u)\right],>\mid u \in U\right\} \\
= & \left\{<u,\left[\left(\vee_{x \in E}\left[\mu_{R}^{l}(u, x) \wedge \gamma_{\sim A}^{l}(x)\right]\right),\left(\vee_{x \in E}\left[\mu_{R}^{r}(u, x) \wedge \gamma_{\sim A}^{r}(x)\right]\right)\right],\right. \\
& {\left.\left[\left(\wedge_{x \in E}\left[\gamma_{R}^{l}(u, x) \vee \mu_{\sim A}^{l}(x)\right]\right),\left(\wedge_{x \in E}\left[\gamma_{R}^{r}(u, x) \vee \mu_{\sim A}^{r}(x)\right]\right)\right]>\mid u \in U\right\}, } \\
= & \left\{<u,\left[\left(\vee_{x \in E}\left[\mu_{R}^{l}(u, x) \wedge \mu_{A}^{l}(x)\right]\right),\left(\vee_{x \in E}\left[\mu_{R}^{r}(u, x) \wedge \mu_{A}^{r}(x)\right]\right)\right],\right. \\
& {\left.\left[\left(\wedge_{x \in E}\left[\gamma_{R}^{l}(u, x) \vee \gamma_{A}^{l}(x)\right]\right),\left(\wedge_{x \in E}\left[\gamma_{R}^{r}(u, x) \vee \gamma_{A}^{r}(x)\right]\right)\right]>\mid u \in U\right\} }
\end{aligned}
$$

$$
\left[\gamma_{\sim A}^{l}(x)=\mu_{A}^{l}(x), \gamma_{\sim A}^{r}(x)=\mu_{A}^{r}(x), \mu_{\sim A}^{l}(x)=\gamma_{A}^{l}(x), \mu_{\sim A}^{r}(x)=\gamma_{A}^{r}(x)\right]
$$

From (1) and $(2) \bar{R}(A)=\sim \underline{R}(\sim A)$.

Similarly, $\underline{R}(A)=\sim \bar{R}(\sim \bar{A})$.

Example 4.5 Let the IVIF soft relation $\mathrm{R}$ be

\begin{tabular}{llll}
\hline $\mathrm{R}$ & $\mathrm{e}_{1}$ & $\mathrm{e}_{2}$ & $\mathrm{e}_{3}$ \\
\hline $\mathrm{u}_{1}$ & {$[0.5,0.7],[0.1,0.3]$} & {$[0,0.1],[0.5,0.8]$} & {$[0.3,0.5],[0.2,0.4]$} \\
$\mathrm{u}_{2}$ & {$[0.1,0.2],[0.4,0.6]$} & {$[0.2,0.5],[0.3,0.4]$} & {$[0.5,0.8],[0.1,0.2]$} \\
$\mathrm{u}_{3}$ & {$[0.1,0.3],[0.4,0.6]$} & {$[0.2,0.3],[0.3,0.6]$} & {$[0,0.1],[0.5,0.8]$} \\
$\mathrm{u}_{4}$ & {$[0.2,0.5],[0.1,0.4]$} & {$[0.3,0.5],[0.2,0.4]$} & {$[0.1,0.2],[0.4,0.7]$} \\
\hline
\end{tabular}

And $\mathrm{A}=\left\{<\mathrm{e}_{1},[0.4,0.6],[0.1,0.3]>,<\mathrm{e}_{2},[0.1,0.4],[0.3,0.6]>,<\mathrm{e}_{3},[0,0.1]\right.$, $[0.5,0.8]>\}$ be the optimal normal decision object.

Then $\sim \mathrm{A}=\left\{\left\langle\mathrm{e}_{1},[0.1,0.3],[0.4,0.6]\right\rangle,\left\langle\mathrm{e}_{2},[0.3,0.6],[0.1,0.4]\right\rangle,<\mathrm{e}_{3},[0.5\right.$, $0.8],[0,0.1]>$.

We show that $\sim \bar{R}(\sim A)=\underline{R}(A)$. 
In Example 3.3 we have

$$
\begin{aligned}
\underline{R}(A) & =\left\{<\mathrm{u}_{1},[0.2,0.4],[0.3,0.5]>,<\mathrm{u}_{2},[0.1,0.2],[0.5,0.8]>,\right. \\
& \left.<\mathrm{u}_{3},[0.3,0.6],[0.2,0.3]>,<\mathrm{u}_{4},[0.2,0.4],[0.3,0.5]>\right\}
\end{aligned}
$$

Now we calculate $\sim \bar{R}(\sim A)$ by the Definition 3.1

Hence $\left.\bar{R}(\sim A)=\left\{<\mathrm{u}_{1},[0.3,0.5],[0.2,0.4]\right\rangle,<\mathrm{u}_{2},[0.5,0.8],[0.1,0.2]\right\rangle,<\mathrm{u}_{3}$, $\left.[0.2,0.3],[0.3,0.6]>,<\mathrm{u}_{4},[0.3,0.5],[0.2,0.4]>\right\}$

Thus $\sim \bar{R}(\sim A)=\left\{<\mathrm{u}_{1},[0.2,0.4],[0.3,0.5]>,<\mathrm{u}_{2},[0.1,0.2],[0.5,0.8]>\right.$,

$$
\left.<\mathrm{u}_{3},[0.3,0.6],[0.2,0.3]>,<\mathrm{u}_{4},[0.2,0.4],[0.3,0.5]>\right\}
$$

From (1) and (2) $\sim \bar{R}(\sim A)=R(A)$.

Similarly $\sim \underline{R}(\sim A)=\bar{R}(A)$.

4.6. Theorem (1) $\underline{R}(A \cap B)=\underline{R}(A) \cap \underline{R}(B)$

(ii) $\bar{R}(A \cup B)=\bar{R}(A) \cup \bar{R}(\bar{B})$

(iii) $\bar{R}(A \cap B) \bar{R}(A) \cap \bar{R}(B)$

(iv) $\underline{R}(A \cup B) \supseteq \underline{R}(A) \cup \underline{R}(B)$

Proof

$$
\underline{R}(A \cap B)=\left\{<u, \mu_{\underline{R}(A \cap B)}(u), \gamma_{\underline{R}(A \cap B)}(u)>\mid u \in U\right\}
$$

$\underline{R}(A \cap B)=\left\{<u,\left[\mu_{\underline{R}(A \cap B)}^{l}(u), \mu_{\underline{R}(A \cap B)}^{r}(u)\right],\left[\gamma_{\underline{R}(A \cap B)}^{l}(u), \gamma_{\underline{R}(A \cap B)}^{r}(u)\right]>\mid u \in U\right\}$

$$
=\left\{<u,\left[\left(\mu_{\underline{R}(A)}^{l}(u) \wedge \mu_{\underline{R}(B)}^{l}(u)\right),\left(\mu_{\underline{R}(A)}^{r}(u) \wedge \mu_{\underline{R}(A)}^{r}(u)\right)\right],\right.
$$$$
\left.\left[\left(\gamma_{\underline{R}(A)}^{l}(u) \wedge \gamma_{\underline{R}(B)}^{l}(u)\right),\left(\gamma_{\underline{R}(A)}^{r}(u) \wedge \gamma_{\underline{R}(A)}^{r}(u)\right)\right]>\mid u \in U\right\}
$$

$=\left\{<u,\left[\left\{\wedge_{x \in E}\left[\gamma_{R}^{l}(u, x) \vee \mu_{A}^{l}(x)\right\} \wedge\left\{\wedge_{x \in E}\left[\gamma_{R}^{l}(u, x) \vee \mu_{B}^{l}(x)\right\},\left\{\wedge_{x \in E}\left[\gamma_{R}^{r}(u, x) \vee \mu_{A}^{r}(x)\right\} \wedge\right.\right.\right.\right.\right.$

$\left\{\wedge_{x \in E}\left[\gamma_{R}^{r}(u, x) \vee \mu_{B}^{r}(x)\right\}\right],\left[\left\{\vee_{x \in E}, \mu_{R}^{l}(u, x) \wedge \gamma_{A}^{l}(x)\right\} \wedge\left\{\vee_{x \in E}\left[\mu_{R}^{l}(u, x) \wedge \gamma_{B}^{l}(x)\right\}\right.\right.$,

$\left\{\vee_{x \in E}\left[\mu_{R}^{r}(u, x) \wedge \gamma_{A}^{r}(x)\right\} \vee\left\{\vee_{x \in E}\left[\mu_{R}^{r}(u, x) \wedge \gamma_{B}^{r}(x)\right\}\right],>\mid u \in U\right\}$

$=\left\{<u,\left[\left\{\wedge_{x \in E}\left[\gamma_{R}^{l}(u, x) \vee \mu_{A}^{l}(x)\right\},\left\{\wedge_{x \in E}\left[\gamma_{R}^{r}(u, x) \vee \mu_{A}^{r}(x)\right\}\right],\left[\left\{\vee_{x \in E}\left[\mu_{R}^{l}(u, x) \wedge \gamma_{A}^{l}(x)\right\}\right.\right.\right.\right.\right.$,

$\left\{\vee_{x \in E}\left[\mu_{R}^{r}(u, x) \wedge \gamma_{A}^{r}(x)\right\}\right] \wedge\left[\left\{\wedge_{x \in E}\left[\gamma_{R}^{l}(u, x) \vee \mu_{B}^{l}(x)\right\},\left\{\wedge_{x \in E}\left[\gamma_{R}^{r}(u, x) \vee\right.\right.\right.\right.$

$\left.\left.\mu_{B}^{r}(x)\right\}\right],\left[\left\{\vee_{x \in E}\left[\mu_{R}^{l}(u, x) \wedge \gamma_{B}^{l}(x)\right\},\left\{\vee_{x \in E}\left[\mu_{R}^{r}(u, x) \wedge \gamma_{B}^{r}(x)\right\}\right]>\mid u \in U\right\}\right.$

$=\{<u, \underline{R}(A) \cap \underline{R}(B)>\mid u \in U\}$.

Here, $\underline{R}(A)=\left[\left\{\wedge_{x \in E}\left[\gamma_{R}^{l}(u, x) \vee \mu_{A}^{l}(x)\right\},\left\{\wedge_{x \in E}\left[\gamma_{R}^{r}(u, x) \vee \mu_{A}^{r}(x)\right\}\right]\right.\right.$,

$$
\left[\left\{\vee_{x \in E}\left[\mu_{R}^{l}(u, x) \wedge \gamma_{A}^{l}(x)\right\},\left\{\vee_{x \in E}\left[\mu_{R}^{r}(u, x) \wedge \gamma_{A}^{r}(x)\right\}\right]\right.\right.
$$

$$
\underline{R}(B)=\left[\left\{\wedge_{x \in E}\left[\gamma_{R}^{l}(u, x) \vee \mu_{B}^{l}(x)\right\},\left\{\wedge_{x \in E}\left[\gamma_{R}^{r}(u, x) \vee \mu_{B}^{r}(x)\right\}\right],\right.\right.
$$

$$
\left[\left\{\vee_{x \in E}\left[\mu_{R}^{l}(u, x) \wedge \gamma_{B}^{l}(x)\right\},\left\{\vee_{x \in E}\left[\mu_{R}^{r}(u, x) \wedge \gamma_{B}^{r}(x)\right\}\right]\right.\right.
$$




$$
\underline{R}(A \cap B)=\underline{R}(A) \cap \underline{R}(B)
$$

Similarly we have $\bar{R}(A \cup B)=\bar{R}(A) \cup \bar{R}(B)$

$$
\begin{gathered}
\bar{R}(A \cap B) \bar{R}(A) \cap \bar{R}(B) \\
\underline{R}(A \cup B) \supseteq \underline{R}(A) \cup \underline{R}(B)
\end{gathered}
$$

\section{Application of IVIF Soft Rough Operators in Determining COVID-19 Patients}

Early detection or prediction is very important to reduce the fatalities of COVID-19 patients. A method using IVIF soft rough operators is used to analyze the identify of Covid-19 patients.

People of all ages can be affected by the new corona virus. Older people and people with pre-existing medical conditions ( such as Asthma, Diabetes, Heart disease, Cancer) appear to be more vulnerable to becoming severely ill with the virus. Most common symptoms are (1) fever (2) dry cough (3) tiredness. Less common symptoms are (a) aches and pains (b) sore throat (c) diarrhea (d) conjunctivitis (e) headache (f) loss of taste or smell $(\mathrm{g})$ a rash on skin or coloration of fingers or toes. Lastly serious symptoms are (i) difficulty breathing or shortness of breath (ii) loss of speech or movement. People seek immediate medical attention if they have serious symptoms. COVID-19 surge is a spread -sheet based tool that hospital administrators and public health officials can use to estimate the surge in demand for hospital based services during the COVID-19 pandemic. A user of COVID-19 surge can produce estimate of the number of COVID-19 patients that need to be hospitalized the number requiring ICU care and the number requiring ventilator support. In this paper we discuss about the people who affected severely in COVID-19. For further study we refer [31, 32].

Suppose that, $U=\{p, q, r, s\}$ is the set of four patients under consideration of a decision maker. Let $\mathrm{E}$ be a parameter set where $\mathrm{E}=\left\{\mathrm{e}_{1}, \mathrm{e}_{2}, \mathrm{e}_{3}\right\}=\{$ fever, dry cough, tiredness $\}$. The doctor wants to locate the COVID- 19 patients with the parameters of E.

Assume that the doctor decides to locate the COVID-19 patients by constructing an IVIF fuzzy soft set $(F, E)$ which is an IVIF soft relation $R$ from $U$ to E. It is presented by the table as in Example 3.3

\begin{tabular}{llll}
\hline $\mathrm{R}$ & $\mathrm{e}_{1}$ & $\mathrm{e}_{2}$ & $\mathrm{e}_{3}$ \\
\hline $\mathrm{p}$ & {$[0.5,0.7],[0.1,0.3]$} & {$[0,0.1],[0.5,0.8]$} & {$[0.3,0.5],[0.2,0.4]$} \\
$\mathrm{q}$ & {$[0.1,0.2],[0.4,0.6]$} & {$[0.2,0.5],[0.3,0.4]$} & {$[0.5,0.8],[0.1,0.2]$} \\
$\mathrm{r}$ & {$[0.1,0.3],[0.4,0.6]$} & {$[0.2,0.3],[0.3,0.6]$} & {$[0,0.1],[0.5,0.8]$} \\
$\mathrm{s}$ & {$[0.2,0.5],[0.1,0.4]$} & {$[0.3,0.5],[0.2,0.4]$} & {$[0.1,0.2],[0.4,0.7]$} \\
\hline
\end{tabular}


Now suppose that the doctor gives the optimum normal decision object $\mathrm{A}$ which is an IVIF subset defined as $\mathrm{A}=\left\{<\mathrm{e}_{1},[0.4,0.6],[0.1,0.3]>,<\mathrm{e}_{2},[0.1,0.4],[0.3,0.6]\right.$ $\left.>,<\mathrm{e}_{3},[0,0.1],[0.5,0.8]>\right\}$.

Then $\bar{R}(A)=\{<\mathrm{p},[0.4,0.6],[0.1,0.3]>,<\mathrm{q},[0.1,0.4],[0.3,0.6]>,<\mathrm{r},[0.1$, $0.3],[0.3,0.6]>,<\mathrm{s},[0.2,0.5],[0.1,0.4]>\}$.

$\& \underline{R}(A)=\{<\mathrm{p},[0.2,0.4],[0.3,0.5]>,<\mathrm{q},[0.1,0.2],[0.5,0.8]>,<\mathrm{r},[0.3,0.6]$, $[0.2,0.3]>,<\mathrm{s},[0.2,0.4],[0.3,0.5]>\}$.

From 4.3, $\bar{R}(A) \oplus \underline{R}(A)=\{<\mathrm{p},[0.52,0.76],[0.03,0.15]>,<\mathrm{q},[0.19,0.52]$, $[0.15,0.48]>,<\mathrm{r},[0.37,0.72],[0.06,0.18]>,<\mathrm{s},[0.36,0.70],[0.03,0.2]>\}$.

Now, according to the experts if the max. of the membership value is $\geq 0.75$ and max. value of the non membership value is $\leq 0.25$ in, $\bar{R}(A) \oplus \underline{R}(A)$ then the patient is suffering from COVID-19. Thus in this example the patient $\mathrm{p}$ is surely effected by COVID-19. The patients $r$ and $s$ should be under observation. The patient $q$ is free from COVID-19.

\section{Conclusion}

In this paper, we have first proposed the concepts of IVIFsoft rough sets. We investigated some properties of IVIF soft rough sets in details. In addition, a new decision method based on IVIFsoft rough sets is proposed. Moreover, practical applications based on IVIF soft rough sets is applied to show its validity. We also believe that the decision making method developed here is expected to attract the researchers working in these areas. Actually, there are at least two aspects in the study of rough set theory: constructive and axiomatic approaches, so is the same to IVIF soft rough sets. So further work should consider the axiomatic approaches to IVIF soft rough sets and the modification of the proposed decision method. In 4.3 , it is to be noted that no algorithms are needed for taking the decision.

Acknowledgements The authors would like to thank the anonymous referees for their valuable comments and suggestions.

Authors' contributions All authors have equal contributions.

Funding Not applicable.

Availability of data and material Not applicable.

Code availability Not applicable.

\section{Declarations}

Conflict of interest Authors declare that they have no conflict of interest.

Ethics approval Data has been collected from reliable souces. We follow all the ethical rules. 


\section{References}

1. Zhou L, Wu WZ (2011) Characterization of rough set approximati in Atanassov intuitionistic fuzzy set theory. Comput Math Appl 62(1):282-296

2. Jiang Y, Tang Y, Chen Q, Liu H, Tang J (2010) Interval-valued intuitionistic fuzzy soft sets and their properties. Comput Math Appl 60:906-918

3. Feng F, Liu X, Leoreanu-Fotea V, Jun YB (2011) Soft sets and soft rough sets. Inf Sci 181(6):1125-1137

4. Kumar S (2020) Monitoring novel corona virus (COVID-19) infections in India by cluster analysis. Ann Data Sci 7:417-425

5. Li J, Guo K, Herrera Viedma E, Lee H, Liu J, Zhong Z, Gomes L, Filip FG, Fang SC, Özdemir MS, Liu XH, Lu G, Sh Y (2020) Culture vs policy: more global collaboration to effectively combat COVID-19. Innovation. https://doi.org/10.1016/j.xinn.2020.100023

6. Liu Y, Gu Z, Xia S, Shi B, Zhou X, Shi Y, Liu J (2020) What are the underlying transmission patterns of COVID-19 outbreak? An age-specific social contact characterization. EClincialMedicine 22:100354-100361

7. Majumder P, Das S, Das R, Tripathy BC (2021) Identification of the most significant risk factor of COVID-19 in economy using cosine similarity measure under SVPNS-environment. Neutrosophic Sets Syst 46:112-127. https://doi.org/10.5281/zenodo.5553497

8. Shi Y, Tian YJ, Kou G, Peng Y, Li JP (2011) Optimization based data mining: theory and applications. Springer, Berlin

9. Bera AK, Jana DK, Banerjee D et al (2021) A two-phase multi-criteria fuzzy group decision making approach for supplier evaluation and order allocation considering multi-objective, multi-product and multi-period. Ann Data Sci 8:577-601

10. Mandal WA (2021) Bipolar pythagorean fuzzy sets and their application in multi attribute decision making problems. Ann Data Sci. https://doi.org/10.1007/s40745-020-00315-8

11. Olson DL, Shi Y (2007) Introduction to business data mining. McGraw-Hill/Irwin, New York

12. Tien JM (2017) Internet of things, real-time decision making, and artificial intelligence. Ann Data Sci 4(2):149-178

13. Wang P (2015) Oriental thinking and fuzzy logic, celebration of the 50th anniversary of fuzzy sets. Ann Data Sci 2:243-244

14. Zadeh LA (1965) Fuzzy sets. Inf Control 8(3):338-353

15. Atanassov KT (1986) Intuitionistic fuzzy sets. Fuzzy Sets Syst 20(1):87-96

16. Gorzałczany MB (1987) A method of inference in approximate reasoning based on interval-valued fuzzy sets. Fuzzy Sets Syst 21(1):1-17

17. Atanassov K, Gargov G (1989) Interval valued intuitionistic fuzzy sets. Fuzzy Sets Syst 31:343-349

18. Pawlak Z (1982) Rough sets. Int J Comput Inf Sci 11(5):145-172

19. Pawlak Z (1991) Rough sets-theoretical aspects to reasoning about data. Kluwer Academic Publisher, Boston

20. Aktaş H, Çağman N (2007) Soft sets and soft groups. Inf Sci 177(13):2726-2735

21. Ali MI, Feng F, Liu X, Min WK, Shabir M (2009) On some new operations in soft set theory. Comput Math Appl 57(9):1547-1553

22. Maji PK, Biswas R, Roy AR (2003) Soft set theory. Comput Math Appl 45(4-5):555-562

23. Molodtsov D (1999) Soft set theory-first results. Comput Math Appl 37(4-5):19-31

24. Molodtsov D (2004) The theory of soft sets. URSS Publishers, Moscow

25. Feng F, Li C, Davvaz B, Ali MI (2010) Soft sets combined with fuzzy sets and rough sets: a tentative approach. Soft Comput 14(9):899-911

26. Maji PK, Biswas R, Roy AR (2001) Fuzzy soft set. J Fuzzy Math 9(3):589-602

27. Majumdar P, Samanta SK (2010) Generalised fuzzy soft sets. Comput Math Appl 59(4):1425-1432

28. Maji PK, Biswas R, Roy AR (2004) Intuitionistic fuzzy soft sets. J Fuzzy Mathematics 12:677-692

29. Park JH, Kim OH, Kwun YC (2012) Some properties of equivalence soft set relations. Comput Math Appl 63(6):1079-1088

30. Zhou L, Wu WZ, Zhang WX (2009) On characterization of intuitionistic fuzzy rough sets based on intuitionistic fuzzy implicators. Inf Sci 179(7):883-898

31. Mukherjee A, Mukherjee A (2020) Application of fuzzy matrix theory in COVID-19 pandemic. IOSR J Math (IOSR-JM) 16:52-56 
32. Mukherjee A, Das R (2020) Neutrosophic bipolar vague soft set and its application to decision making problems. Neutrosophic Sets Syst 32:410-424

Publisher's Note Springer Nature remains neutral with regard to jurisdictional claims in published maps and institutional affiliations. 